\title{
The Migraine-Ischemic Stroke Relation in Young Adults
}

\author{
Alessandro Pezzini, ${ }^{1}$ Elisabetta Del Zotto, ${ }^{2}$ Alessia Giossi, ${ }^{1}$ Irene Volonghi, ${ }^{1}$ \\ Paolo Costa, ${ }^{1}$ Giorgio Dalla Volta, ${ }^{3}$ and Alessandro Padovani ${ }^{1}$ \\ ${ }^{1}$ Dipartimento di Scienze Mediche e Chirurgiche, Clinica Neurologica, Università degli Studi di Brescia, \\ Piazzale Spedali Civili, 1, 25100 Brescia, Italy \\ ${ }^{2}$ Dipartimento di Scienze Biomediche e Biotecnologie, Università degli Studi di Brescia, 25100 Brescia, Italy \\ ${ }^{3}$ Unità di Neurologia, Istituto Clinico Città di Brescia, 25123 Brescia, Italy \\ Correspondence should be addressed to Alessandro Pezzini, ale_pezzini@hotmail.com
}

Received 19 September 2010; Accepted 22 November 2010

Academic Editor: Halvor Naess

Copyright (C 2011 Alessandro Pezzini et al. This is an open access article distributed under the Creative Commons Attribution License, which permits unrestricted use, distribution, and reproduction in any medium, provided the original work is properly cited.

In spite of the strong epidemiologic evidence linking migraine and ischemic stroke in young adults, the mechanisms explaining this association remain poorly understood. The observation that stroke occurs more frequently during the interictal phase of migraine prompts to speculation that an indirect relation between the two diseases might exist. In this regard, four major issues might be considered which may be summarized as follows: (1) the migraine-ischemic stroke relation is influenced by specific risk factors such as patent foramen ovale or endothelial dysfunction and more frequent in particular conditions like spontaneous cervical artery dissection; (2) migraine is associated with an increased prevalence of cardiovascular risk factors; (3) the link is caused by migraine-specific drugs; (4) migraine and ischemic vascular events are linked via a genetic component. In the present paper, we will review epidemiological studies, discuss potential mechanisms of migraine-induced stroke and comorbid ischemic stroke, and pose new research questions.

\section{Introduction}

Migraine affects about $15 \%$ of people in developed countries [1] and is three times more common in women than in men. Patients have a median of one attack per month, and $25 \%$ have at least two attacks per month [2]. Patients with more than one attack per month are at increased risk of brain lesions [3]. In the last decades, several studies have emphasized the high prevalence of migraine among young individuals with stroke as well as a dysfunction of cerebral arteries during migraine attacks and the finding of silent infarct-like brain lesions in migraineurs, thus leading to the hypothesis that a comorbidity between migraine and cerebral ischemia exists [4].

\section{Evidence of Association}

The first epidemiological suggestion that migraine may be an independent risk factor for stroke came from the Collaborative Group for the Study of Stroke in Young Women, published in 1975, which showed an increased relative risk of stroke with migraine compared with neighbor controls [5]. Since then, the association of migraine with the risk of stroke has been investigated in several observational studies, most of which have been summarized in a recent meta-analysis [6].

According to this meta-analysis, the pooled relative risk of ischemic stroke among patients with any type of migraine is 1.73 (95\% CI, 1.31 to 2.29 ). The relative risk for women is increased (RR 2.08, 95\% CI 1.13 to 3.84) but not for men (RR $1.37,95 \%$ CI 0.89 to 2.11 ). Stratifying analysis by age, people with migraine aged less than 45 have an higher risk than the overall group (RR 2.65, 95\% CI 1.41 to 4.97 ), which was further increased among young women (RR 3.95, 95\% CI 2.21 to 6.04 ).

The risk is apparently more than tripled by smoking (RR 9.09, 95\% CI 4.22 to 19.34 ) and more pronounced by oral contraceptives (OCs) use (RR 7.02, 95\% CI 1.51 to 32.68). In line with this analysis, data from the Stroke Prevention in Young Women study (SPYW) showed a higher risk of stroke in women with probable migraine with visual aura who were cigarette smokers and oral contraceptive users 
[7]. Overall, these observations reinforced the hypothesis that specific subgroups of patients in which the migrainestroke pathogenic link is more expressed might be identified. Moreover, the meta-analysis found an increased risk of ischemic stroke among people who had migraine with aura (MA) (RR 2.16, 95\% CI, 1.53 to 3.03) but not among those who had migraine without aura (MO) (RR 1.23, 95\% CI 0.90 to 1.69), challenging whether MO should be considered a stroke risk factor. Is this enough to conclude that migraine is a risk factor for stroke? Some limitations of the included studies should be considered. First, migraine is biologically heterogeneous [8], and latent class analysis of migraine symptoms indicated the existence of a continuum of severity, with MA being more severe but not etiologically distinct from MO, thus reinforcing the view that the two migraine subtypes are not separate entities $[9,10]$. The use of the categories of the International Headache Society classification [11] as phenotypes (MO and MA) might present an oversimplified picture of migraine phenotype, which in reality has a complex genetic and environmental background, and using this phenotype might result in too heterogeneous a set of patients for association analyses. As in other complex diseases, the use of single traits or other new phenotyping strategies for migraine might help in stratifying study samples into less heterogeneous groups. Second, potential bias in the selection of patients should be taken into account. At least theoretically, a referral bias may exist if stroke patients with migraine would be referred to the recruiting centres more frequently than stroke cases without migraine, or if the investigators were more prone to include stroke cases with migraine than without migraine. A further selection bias could be the consequence of a stroke-migraine misdiagnosis. As TIA are sometimes difficult to distinguish from an attack of MA, especially when the aura occurs without headache, and migraine with prolonged neurological aura (lasting longer than 24 hours) may mimic stroke, the end results of such misclassifications would be an overestimation of the prevalence of migraine in cases and, therefore, an overestimation of the risk. Third, in most of the considered studies, a consistent definitions for migraine is lacking. Accurate diagnosis of migraine is important to avoid nondifferential misclassification of exposure, which will bias the risk estimate towards showing no association. If this is the case, however, we cannot but assume that the increased risk of stroke emerging from the pooled analysis of data from observational studies is rather an underestimation of the effect. As such, it should be retained as an argument in favour of the reported association between migraine and stroke. Furthermore, in case-control studies, an interviewer bias and a recall bias can arise as possible consequences of the retrospective design. Fourth, in some of the studies, the influence of several confounders on the final results was not considered, while others were not controlled for. These include, for example, the use of medications with a potential effect on stroke risk (i.e., antihypertensive agents), or risk factors for both migraine and stroke, such as antiphospholipids antibodies. Finally, most studies are limited to younger individuals (aged 45 years or younger) leaving the association between migraine and stroke among the elderly unclear and ignoring the fact that migraine may start later in life.

Overall, there is strong epidemiological evidence that migraine, particularly MA, is associated with increased risk of cerebral ischemic events, which appears to be stronger among the young but may persist in the elderly. In this regard, data from 39,876 US female health professionals aged 45 or older included in the Women's Health study after a mean of 9 years of followup showed that MA increased 1.5-fold the risk of total stroke after adjusting for potential confounders of age, hypertension, menopausal status, contraceptive use, and alcohol consumption (HR $1.53 ; 95 \%$ CI 1.02 to 2.31 ) and a 1.7 -fold the risk of ischemic stroke (HR 1.71; 95\% CI 1.11 to 2.66 ) when compared to participants without migraine [12]. In contrast, there was no association between $\mathrm{MO}$ and total stroke or ischemic stroke. This association between MA and cerebrovascular disease as well as with ischemic stroke was confirmed in a large cohort of individuals $\geq 55$ years of age at the time of migraine assessment, who participated to the Atherosclerosis Risk in Communities (ARICs) study [13]. A recent population-based cohort study during up to four decades of followup found that at midlife people with MA were at increased risk of all-cause mortality (HR 1.21, 95\% CI 1.12 to 1.30 ) and mortality from cardiovascular disease (HR $1.27,95 \%$ CI 1.13 to 1.43 ) compared with people with no headache, while those with MO and nonmigraine headache were not. In particular, people with MA were at increased risk of mortality from coronary heart disease (HR 1.28, 95\% CI 1.11 to 1.49 ) and stroke (HR 1.40, 95\% CI 1.10 to 1.78) [14]. Thus, these findings provide arguments to the assumption that migraine may have some influence on stroke risk even in older subgroups. However, effect modification by age is evident from prospective data, the risk of stroke being higher in younger age groups and decreasing over time, with no increased risk among the elderly (age 60 or older) according to case-control analyses [15].

\section{What Is the Pathogenic Mechanism Linking Migraine and Stroke?}

Although the relationship between migraine and stroke remains one of the most perplexing problems for neurologists, there are also valid arguments to assume that the hypothesis of a pathogenic relation between these two entities is biologically plausible. The observation that stroke may occur during migraine attacks prompts to speculation that migraine may directly cause an ischemic event (i.e., migrainous infarct). Alternatively, as stroke occurs more frequently during the interictal phase of migraine, an indirect relation between the two diseases might exist. In this regard, four major issues might be considered which may be summarized as follows: (1) migraine-ischemic stroke pathway is modulated by the intervention of common risk factors; (2) migraine is associated with an increased prevalence of cardiovascular risk factors; (3) the link is caused by migrainespecific drugs; (4) migraine and ischemic vascular events are linked via a genetic component [16]. 


\section{Is Migraine a Direct Cause of Cerebral Ischemia?}

"Migrainous infarction" is defined as a stroke occurring during a typical attack of MA in the IHS migraine classification. This condition suggests a causal relationship between stroke and migraine. Diagnostic criteria include a history of MA and the neurological deficits occurring in the same vascular distribution as the aura, persisting for more than 60 minutes, and being associated with an ischemic brain lesion in a suitable territory demonstrated by neuroimaging. Other possible causes of ischemic stroke have to be excluded by appropriate investigations [11]. However, which investigations should be done and when is not clear. The absence of causes other than migraine does not necessarily imply that migraine is the cause, given that about half of the ischemic strokes in young adults have no detectable cause. According to large series, the incidence of migrainous infarction [17-21] varies between 0.5 and $1.5 \%$ of all ischemic strokes and 10 and $14 \%$ of ischemic strokes in young patients. The clinical features typifying migrainous stroke included female sex, mean age in the low-to-mid-30 s, a history of cigarette smoking, and ischemic involvement of the PCA territory [19]. Although the limitations inherent in the diagnostic criteria which might be too strict for a correct diagnosis of migrainous infarction, and in the consequent weakness of the epidemiological studies, it seems reasonable to assume that migrainous infarction does not account for all strokes occurring during migraine attacks, and, overall, it is responsible for only a minority of migraine-related infarcts. Notwithstanding, it represents a useful model to understand the potential mechanisms underlying the relation between migraine and stroke. Migraine is considered to be a neurovascular disorder, in which arterial constriction and decreased blood flow to the posterior circulation are consequences of a spreading wave of neuronal depression in the cerebral cortex (cortical spreading depression, CSD). The first vascular phenomenon associated to CSD is a short-lived increases in cerebral blood flow (CBF) and tissue hyperoxia [22] followed by a more profound oligemia and consequent increased intraparenchymal vascular resistance [23]. Thus, low flow in major intracerebral vessels may be due to increased downstream resistance, not major intracranial arterial vasospasm. Essentially, a prolonged decreased in $\mathrm{CBF}$ and neuronally mediated vasodilatation could cause sluggish flow in large intracerebral vessels during the aura of migraine. The combined effect of conditions predisposing to coagulopathy, such as dehydration hyperviscosity or intravascular thrombosis, could induce migraine-induced cerebral infarction, although rarely. Neurogenically mediated inflammatory responses accompanying vasodilation of extra-parenchymal vessels caused by release of vasoactive peptides, nitric oxide (NO), activation of cytokines, and upregulation of adhesion molecules also predispose to intravascular thrombosis [24]. This could explain why migraine-induced stroke usually respects intracranial arterial territories while aura involves more widespread brain regions. In addition, frequent aura, if due to CSD, could induce cytotoxic cell damage and gliosis based on glutamate release or excess intracellular calcium accumulation [25]. Thus, a persistent neurological deficit could be due to selective neuronal necrosis. Finally, the occurrence of arterial vasospasm, as a consequence of the release of vasoconstrictive substances such as endothelin and serotonin, once thought to be the mechanism of migraine aura, has been implicated in migrainous infarction, although documented cases are rare.

\section{Is Migraine an Indirect Cause of Cerebral Ischemia?}

\subsection{Potential Common Biologic Mechanisms}

5.1.1. Patent Foramen Ovale. Several investigators have reported an increased prevalence of patent foramen ovale (PFO), an interatrial septal cardiac abnormality associated with increased risk of ischemic stroke in young adults in patients who suffered MA than in patients without migraine $[26,27]$. Similarly, in patients with ischemic stroke, MA is twice as prevalent in patients with PFO than in those without $[28,29]$. Multiple observational studies, from both single and multicentre experiences, suggest PFO closure to reduce the frequency of migraine attacks. In particular, among migraineurs, this might be proposed for those patients in the MA subgroup and might indirectly reduce the risk of stroke, in spite of the small stroke predisposing effect of PFO and some recent findings indicating no stronger association between MA and ischemic stroke among women with PFO compared with women without [7]. However, the following limitations in these reports need to be considered including absence of control group, retrospective design which implicates recall bias, placebo effect that can result in up to $70 \%$ reduction of attacks frequencies $[30,31]$, administration of aspirin after PFO closure, and its potential prophylactic effect [32]. Paradoxical embolism is suggested to be the causal link between migraine and PFO, but insufficient data are available to substantiate the hypothesis that migraine frequency (and, indirectly, ischemic stroke risk) is reduced by PFO closure. The only way to address this issue is by randomization. At present, only one prospective, randomised, double-blind trial on the therapeutic effect of PFO device closure on MA patients compared to sham has been conducted (Migraine Intervention with STARFlex Technology, MIST) without reaching a positive end point and being associated with substantial controversy [33, 34]. A more comprehensive analysis of current data is desirable to provide information about how to identify patients who may have an improvement of their migraine, and a large number of patients with longer followup seems necessary. Other studies examining the efficacy of PFO closure for prevention of migraine are under way. Based on all these findings, the possibility of a PFO-migraine-ischemic stroke triangular association remains actually a matter of speculation.

5.1.2. Endothelial Dysfunction. Another topic of recent attention is that migraine may be a risk factor for endothelial dysfunction, representing a challenging link to ischemic stroke and heart disease. Endothelial dysfunction is characterized 
by reduction in bioavailability of vasodilator (such as NO), increase in endothelial-derived contracting factors, and consequent impairment of the reactivity of the microvasculature. It also comprises endothelial activation, characterized by a procoagulant, proinflammatory, and proliferative milieu, which, in turns, predisposes to an increased rate of cerebroand cardiovascular ischemic events. Traditional vascular risk factors are known to have an unfavorable effect on endothelial function. Therefore, given its biological properties, vascular endothelium (and, thus, endothelial dysfunction) can be considered as the "missing link" between any risk factor and its detrimental vascular effect. In this context, endothelial dysfunction can be regarded as "the ultimate risk of the risk factors" [35]. Endothelial dysfunction is mediated by increased oxidative stress, an important promotor of the inflammatory process [35], which has been proposed in the pathogenesis of migraine. In fact, compared to migrainefree controls, oxidative stress markers have been found to be higher in migraineurs, even during the interictal period, thus yielding support to the association. These findings, as well as the known efficacy of anti-inflammatory agents in migraine attacks, indirectly support this assumption and further strengthen the hypothesis of a pathogenic relation migraine-endothelial dysfunction-cerebral ischemia.

5.1.3. Cervical Artery Dissection. In the last years, migraine has been suggested to be a predisposing condition for spontaneous cervical artery dissections (sCADs), one of the most common causes of stroke in young patients leading to speculation that migraine might be a predisposing condition for specific pathogenic subtypes of ischemic stroke particularly in young patients. In two French case-control studies, migraine resulted twice as common in patients with sCAD than in patients whose ischemic stroke was not related to an sCAD $[36,37]$, and this association was stronger and more significant in patients with dissections involving multiple vessels. A large Italian case-control study confirmed these findings [38]. The association between SCAD and a specific migraine subtype is controversial since it has been suggested to be stronger for MO than for MA, but recently a hospital-based case-control study found a higher prevalence of migraine with aura among $\mathrm{SCAD}$ patients as compared to a control group [39].

The mechanism by which migraine may affect the risk of sCAD is unknown. A common generalized vascular disorder is hypothesized to be a predisposing condition for both diseases. Increased activity of serum elastase, a metallopeptidase which degrades specific elastin-type aminoacid sequences, reported in migraineurs suggests a possible extracellular matrix degradation [40] which might facilitate sCAD occurrence. Furthermore, in line with previous observations of altered common carotid artery distensibility in patients with sCAD [41], Lucas and coworkers recently reported that the endothelium-dependent vasodilatation assessed in the brachial artery is significantly impaired in these subjects [42]. Similar vascular changes have been observed in migraine patients during interictal periods [43] and replicated in a recent cross-sectional study in migraineurs of recent onset, thus excluding possibility of bias due to longstanding history of migraine and repeated exposure to vasoconstrictor drugs [44]. Finally, the analysis of small families has shown that the structural abnormalities related to sCAD might be familial and follow an autosomal dominant pattern of inheritance $[45,46]$. This implicates that genetically determined alterations of the extracellular matrix may play a crucial pathogenic role and that candidate genes involved in the regulation of the endothelial and the vessel wall functions, might increase susceptibility to both conditions [47-49].

\section{Migraine and Cardiovascular Risk Factors}

Several reports have associated migraine with a more unfavourable cardiovascular risk profile. According to the results of the Genetic Epidemiology of Migraine (GEM) study, migraineurs are more likely to smoke and to have a parental history of myocardial infarction. MA was also found to be associated with an unfavourable cholesterol profile, elevated blood pressure, early onset coronary heart disease, or stroke [50]. Data from the population-based American Migraine Prevalence and Prevention (AMPP) study recently provided further support to the perception that migraineurs have a higher probability of having an unfavorable risk factors profile [51]. However, a number of large-scale epidemiologic analyses conducted over the last years have questioned these findings and suggested that the migraine-stroke association is particularly present in the absence of traditional cardiovascular risk factors [16, 52]. Therefore, the possibility that the relation between migraine and ischemic stroke may be due to the known pathogenic effect of traditional vascular risk factors seems unlikely.

\section{Effects of Migraine-Specific Drugs}

Since the potential of triptans and ergotamine for adverse vascular effects, in the last years large-scaled studies have investigated the risk of ischemic events and death in migraineurs patients treated with these agents. Data from General Practice Research Database in the United Kingdom showed that in general practice triptan treatment did not increase the risk of ischemic events [53]. Similarly, this finding was confirmed by a wide retrospective cohort study from a health care provider in USA [54]. This study also investigated the rates of vascular events in relation to ergotamine use finding no association. Recently, a retrospective nested case-control study using data from the PHARMO Record Linkage System conducted in Netherlands investigated whether overuse of triptans and ergotamina is associated with an increased risk of ischemic events [55]. Results showed that while ergotamine overuse increases significantly the risk of ischemic complications (OR 2.55; 95\% CI, 1.22-5.36) especially in patients concomitantly using cardiovascular drugs (OR 8.52; 95\% CI, 2.57-28.2), overuse of triptan, neither in the general populations nor in those using cardiovascular drugs increases the risk of cerebral, cardiovascular, or peripheral ischemic events. However, therapeutic doses of either triptans or ergotamines were not associated with an increased risk of ischemic vascular events. 
Taken together, these findings suggest that triptan use and even triptan overuse are generally safe although heighten the risk of ischemic complications due to ergotamine overuse, likely in relation to its greater vasoconstrictive properties.

\section{Genetic Influence}

Based on twin and family history studies, genetic predisposition has been suggested to play a major role in the occurrence of both migraine and ischemic stroke. Interestingly, several candidate genes for migraine are also good candidate for cerebral ischemia. Among them, in spite of the inconsistent in migraine [56], the C677T polymorphism of the MTHFR gene seems particularly promising, because of its probable independent effect on ischemic stroke risk [57]. In this regard, recently the results of a genotype-migraine-stroke interaction study have been reported in which the TTgenotype of the C677T MTHFR polymorphism was found to be associated to both diseases and influence their relation [58]. Applying a mediation modelling strategy on a group of patients with $\mathrm{SCAD}$, a group of young patients whose ischemic stroke was unrelated to an SCAD (non-CAD) and a group of control subjects, both migraine and the TTgenotype were found significantly associated to the group of patients with ischemic stroke as compared to controls, with a stronger stroke subtype specific effect for sCAD. These findings suggest that migraine may act as mediator in the MTHFR-ischemic stroke pathway with a more prominent effect in the subgroup of patients with sCAD and prompt speculating that the C677T MTHFR polymorphism may be one of the hitherto unknown factors linking migraine to cerebral ischemia. These findings have been replicated in a separate analysis of the Women's Health study data [59]. Overall, these findings may be viewed considering the prevailing hypothesis that migraine and ischemic stroke are the end phenotype of polygenic disorders reflecting the effect of several genetic loci modulating different pathophysiological processes and the combination of many hundreds of genetic variants; as a consequence, it is reasonable to hypothesize that certain genes might have an effect on both diseases and influence their relation. At what levels in the migrainestroke pathway these genetic influences might be operating to increase the propensity to cerebral ischemia and whether such effects might vary according to different stroke subtypes are important and still poorly investigated aspects of stroke pathogenesis.

Ischemic stroke and migraine further coexist in the context of some syndrome characterized by peculiar phenotype, proven inherited background, and chronic alterations of the wall of cerebral small vessel arteries suggesting a common pathogenic mechanism shared by these two conditions.

CADASIL is an autosomal dominant disease of vascular and smooth muscle cells due to Notch-3 mutations [60], characterized by leucoencephalopathy, small deep infarcts, and subcortical dementia. MA is usually the first manifestation, presenting about 15 years before stroke and before the appearance of MRI signal abnormalities. MA is present in one-third of symptomatic subjects, and its frequency can vary greatly among the affected pedigrees. In $40 \%$ of families, more than $60 \%$ of symptomatic subjects had a history of MA [61, 62] and within some families, MA is the most important clinical aspect of the phenotype. The mechanism underlying MA in CADASIL is not clear. Presenting 1020 years before ischemic manifestations, MA is not the consequence of subcortical infarcts. In CADASIL, absence of difference in the frequency and distribution of whitematter abnormalities between patients with and without MA suggests that chronic subcortical hypoperfusion is also unlikely. Another hypothesis is that MA directly relates to dysfunction of smooth muscle cells of meningeal and cortical vessels, triggering CSD [63]. Furthermore, if the cells signalling abnormalities (resulting from the mutation) extend and reach neurons, the resulting hyperexcitable membrane instability could predispose to CSD.

Cerebroretinal vasculopathy (CRV) and hereditary endotheliopathy with retinopathy, nephropathy, and stroke (HERNS) are two rare inherited conditions characterized by a primary microangiopathy of the brain in combination with vascular retinopathy.

Mitochondrial myopathy, encephalopathy, lactic acidosis, and stroke-like episodes (MELAS) is associated to several mutations in mitochondrial DNA (mtDNA). The phenotypic expression is highly variable ranging from asymptomatic state to severe childhood multisystem disease with lactic acidosis. Recurrent episodes of headache (mostly migraine) are part of the clinical spectrum.

Migraine is also part of the clinical spectrum of hereditary haemorragic teleangiectasia (HHT; Osler-WeberRendu disease), an autosomal dominant vascular dysplasia characterized by a high prevalence of vascular malformations in various organs, including lung, liver, kidney, and brain, as well as by mucocutaneous teleangiectasias [64].

\section{Conclusion}

Given all the epidemiological evidence suggesting that migraine, in particular MA, increases the risk of cerebral ischemic events, some questions related to the pathogenic link between migraine and ischemic stroke still remain. Strong arguments support the assumption that MA is associated with a systemic vascular disorder, especially in young stroke patients. The use of new phenotyping strategies for migraine and ischemic stroke should be taken into account in order to provide a more direct insight into the underlying biology of the two diseases and how to identify migraineurs at highest risk of ischemic stroke. Whether stroke can be prevented by migraine prophylaxis, endothelial repair, antiinflammatory treatment, platelet inhibition, or a combination of these strategies is a further goal of future research.

\section{References}

[1] L. J. Stovner, J. A. Zwart, K. Hagen, G. M. Terwindt, and J. Pascual, "Epidemiology of headache in Europe," European Journal of Neurology, vol. 13, no. 4, pp. 333-345, 2006.

[2] L. J. Launer, G. M. Terwindt, and M. D. Ferrari, "The prevalence and characteristics of migraine in a populationbased cohort: the GEM study," Neurology, vol. 53, no. 3, pp. 537-542, 1999. 
[3] M. C. Kruit, M. A. van Buchem, P. A. M. Hofman et al., "Migraine as a risk factor for subclinical brain lesions," Journal of the American Medical Association, vol. 291, no. 4, pp. 427434, 2004.

[4] R. B. Lipton and S. D. Silberstein, "Why study the comorbidity of migraine?” Neurology, vol. 44, no. 10, supplement 7, pp. S4S5, 1994.

[5] Collaborative Group of the Study of Stroke in Young Women, "Oral contraceptives and stroke in young women. Associated risk factors," Journal of the American Medical Association, vol. 231, no. 7, pp. 718-722, 1975.

[6] M. Schürks, P. M. Rist, M. E. Bigal, J. E. Buring, R. B. Lipton, and T. Kurth, "Migraine and cardiovascular disease: systematic review and meta-analysis," BMJ, vol. 339, no. 7728, p. 1015, 2009.

[7] L. R. MacClellan, W. Giles, J. Cole et al., "Probable migraine with visual aura and risk of ischemic stroke: the stroke prevention in young women study," Stroke, vol. 38, no. 9, pp. 2438-2445, 2007.

[8] E. J. Mulder, C. Van Baal, D. Gaist et al., "Genetic and environmental influences on migraine: a twin study across six countries," Twin Research, vol. 6, no. 5, pp. 422-431, 2003.

[9] D. R. Nyholt, N. G. Gillespie, A. C. Heath, K. R. Merikangas, D. L. Duffy, and N. G. Martin, "Latent class and genetic analysis does not support migraine with aura and migraine without aura as separate entities," Genetic Epidemiology, vol. 26, no. 3, pp. 231-244, 2004.

[10] R. A. Lea, D. R. Nyholt, R. P. Curtain et al., "A genome-wide scan provides evidence for loci influencing a severe heritable form of common migraine," Neurogenetics, vol. 6, no. 2, pp. 67-72, 2005.

[11] Headache Classification Subcommittee of the International Headache Society, "The international classification of headache disorders," Cephalalgia, vol. 24, supplement 1, pp. 24-36, 2004.

[12] T. Kurth, M. A. Slomke, C. S. Kase et al., "Migraine, headache, and the risk of stroke in women: a prospective study," Neurology, vol. 64, no. 6, pp. 1020-1026, 2005.

[13] P. E. Stang, A. P. Carson, K. M. Rose et al., "Headache, cerebrovascular symptoms, and stroke: the atherosclerosis risk in communities study," Neurology, vol. 64, no. 9, pp. 15731577, 2005.

[14] L. S. Gudmundsson, A. I. Scher, T. Aspelund et al., "Migraine with aura and risk of cardiovascular and all cause mortality in men and women: prospective cohort study," BMJ, vol. 341, p. c3966, 2010.

[15] A. Mosek, R. Marom, A. D. Korczyn, and N. Bornstein, "A history of migraine is not a risk factor to develop an ischemic stroke in the elderly," Headache, vol. 41, no. 4, pp. 399-401, 2001.

[16] T. Kurth, "Migraine and ischaemic vascular events," Cephalalgia, vol. 27, no. 8, pp. 967-975, 2007.

[17] K. R. Merikangas, B. T. Fenton, S. H. Cheng, M. J. Stolar, and $\mathrm{N}$. Risch, "Association between migraine and stroke in a largescale epidemiological study of the United States," Archives of Neurology, vol. 54, no. 4, pp. 362-368, 1997.

[18] E. Linetsky, R. R. Leker, and T. Ben-Hur, "Headache characteristics in patients after migrainous stroke," Neurology, vol. 57, no. 1, pp. 130-132, 2001.

[19] A. Arboix, J. Massons, L. García-Eroles, M. Oliveres, M. Balcells, and C. Targa, "Migrainous cerebral infarction in the Sagrat Cor Hospital of Barcelona Stroke Registry," Cephalalgia, vol. 23, no. 5, pp. 389-394, 2003.
[20] S. J. Kittner, B. J. Stern, M. Wozniak et al., "Cerebral infarction in young adults: the Baltimore-Washington Cooperative Young Stroke Study," Neurology, vol. 50, no. 4, pp. 890-894, 1998.

[21] D. Sochurkova, T. Moreau, M. Lemesle, M. Menassa, M. Giroud, and R. Dumas, "Migraine history and migraineinduced stroke in the Dijon Stroke Registry," Neuroepidemiology, vol. 18, no. 2, pp. 85-91, 1999.

[22] Y. Cao, K. M. A. Welch, S. Aurora, and E. M. Vikingstad, "Functional MRI-BOLD of visually triggered headache in patients with migraine," Archives of Neurology, vol. 56, no. 5, pp. 548-554, 1999.

[23] F. M. Cutrer, A. G. Sorensen, R. M. Weisskoff et al., "Perfusionweighted imaging defects during spontaneous migrainous aura," Annals of Neurology, vol. 43, no. 1, pp. 25-31, 1998.

[24] H. Bolay, U. Reuter, A. K. Dunn, Z. Huang, D. A. Boas, and M. A. Moskowitz, "Intrinsic brain activity triggers trigeminal meningeal afferents in a migraine model," Nature Medicine, vol. 8, no. 2, pp. 136-142, 2002.

[25] K. M. A. Welch and N. M. Ramadan, "Mitochondria, magnesium and migraine," Journal of the Neurological Sciences, vol. 134, no. 1-2, pp. 9-14, 1995.

[26] G. P. Anzola, M. Magoni, M. Guindani, L. Rozzini, and G. D. Volta, "Potential source of cerebral embolism in migraine with aura: a transcranial Doppler study," Neurology, vol. 52, no. 8, pp. 1622-1625, 1999.

[27] M. Del Sette, S. Angeli, M. Leandri et al., "Migraine with aura and right-to-left shunt on transcranial Doppler: a case-control study," Cerebrovascular Diseases, vol. 8, no. 6, pp. 327-330, 1998.

[28] C. Lamy, C. Giannesini, M. Zuber et al., "Clinical and imaging findings in cryptogenic stroke patients with and without patent foramen ovale: the PFO-ASA study," Stroke, vol. 33, no. 3, pp. 706-711, 2002.

[29] R. Sztajzel, D. Genoud, S. Roth, B. Mermillod, and J. Le Floch-Rohr, "Patent foramen ovale, a possible cause of symptomatic migraine: a study of 74 patients with acute ischemic stroke," Cerebrovascular Diseases, vol. 13, no. 2, pp. 102-106, 2002.

[30] H. J. Gelmers, P. Henry, J. Lucas et al., "European multicenter trial of Nimodipine in the prophylaxis of common migraine (migraine without aura)," Headache, vol. 29, no. 10, pp. 633638, 1989.

[31] H. J. Gelmers, P. Henry, J. Lucas et al., "European multicenter trial of Nimodipine in the prophylaxis of classic migraine (migraine with aura)," Headache, vol. 29, no. 10, pp. 639-642, 1989.

[32] H.-C. Diener, E. Hartung, J. Chrubasik et al., "A comparative study of oral acetylsalicyclic acid and metoprolol for the prophylactic treatment of migraine. A randomized, controlled, double-blind, parallel group phase III study," Cephalalgia, vol. 21, no. 2, pp. 120-128, 2001.

[33] A. Dowson, M. J. Mullen, R. Peatfield et al., "Migraine Intervention with STARFlex Technology (MIST) trial: a prospective, multicenter, double-blind, sham-controlled trial to evaluate the effectiveness of patent foramen ovale closure with STARFlex septal repair implant to resolve refractory migraine headache," Circulation, vol. 117, no. 11, pp. 13971404, 2008.

[34] S. J. Tepper, F. D. Sheftell, and M. E. Bigal, "The patent foramen ovale-migraine question," Neurological Sciences, vol. 28, no. 2, pp. S118-S123, 2007. 
[35] P. O. Bonetti, L. O. Lerman, and A. Lerman, "Endothelial dysfunction: a marker of atherosclerotic risk," Arteriosclerosis, Thrombosis, and Vascular Biology, vol. 23, no. 2, pp. 168-175, 2003.

[36] J. D’Anglejan-Chatillon, V. Ribeiro, J. L. Mas, B. D. Youl, and M. G. Bousser, "Migraine: a risk factor for dissection of cervical arteries," Headache, vol. 29, no. 9, pp. 560-561, 1989.

[37] C. Tzourio, L. Benslamia, B. Guillon et al., "Migraine and the risk of cervical artery dissection: a case-control study," Neurology, vol. 59, no. 3, pp. 435-437, 2002.

[38] A. Pezzini, F. Granella, M. Grassi et al., "History of migraine and the risk of spontaneous cervical artery dissection," Cephalalgia, vol. 25, no. 8, pp. 575-580, 2005.

[39] V. Artto, T. M. Metso, A. J. Metso et al., "Migraine with aura is a risk factor for cervical artery dissection: a case-control study," Cerebrovascular Diseases, vol. 30, no. 1, pp. 36-40, 2010.

[40] C. Tzourio, M. El Amrani, L. Robert, and A. Alpérovitch, "Serum elastase activity is elevated in migraine," Annals of Neurology, vol. 47, no. 5, pp. 648-651, 2000.

[41] B. Guillon, C. Tzourio, V. Biousse, V. Adraï, M. G. Bousser, and P. J. Touboul, "Arterial wall properties in carotid artery dissection: an ultrasound study," Neurology, vol. 55, no. 5, pp. 663-666, 2000.

[42] C. Lucas, J. L. Lecroart, C. Gautier et al., "Impairment of endothelial function in patients with spontaneous cervical artery dissection: evidence for a general arterial wall disease," Cerebrovascular Diseases, vol. 17, no. 2-3, pp. 170-174, 2004.

[43] J. de Hoon, J. M. Willigers, J. Troost, H. A. Struijker-Boudier, and L. M. Van Bortel, "Cranial and peripheral interictal vascular changes in migraine patients," Cephalalgia, vol. 23, no. 2, pp. 96-104, 2003.

[44] F. H. Vanmolkot, L. M. van Bortel, and J. N. de Hoon, "Altered arterial function in migraine of recent onset," Neurology, vol. 68, no. 19, pp. 1563-1570, 2007.

[45] C. Grond-Ginsbach, B. Klima, R. Weber et al., "Exclusion mapping of the genetic predisposition for cervical artery dissections by linkage analysis," Annals of Neurology, vol. 52, no. 3, pp. 359-364, 2002.

[46] I. Hausser, U. Müller, S. Engelter et al., "Different types of connective tissue alterations associated with cervical artery dissections," Acta Neuropathologica, vol. 107, no. 6, pp. 509514, 2004

[47] M. Kloss, T. Wiest, S. Hyrenbach et al., "MTHFR 677TT genotype increases the risk for cervical artery dissections," Journal of Neurology, Neurosurgery and Psychiatry, vol. 77, no. 8, pp. 951-952, 2006.

[48] A. Pezzini, E. Del Zotto, S. Archetti et al., "Plasma homocysteine concentration, C677T MTHFR genotype, and 844ins68bp CBS genotype in young adults with spontaneous cervical artery dissection and atherothrombotic stroke," Stroke, vol. 33, no. 3, pp. 664-669, 2002.

[49] A. I. Scher, G. M. Terwindt, W. M. M. Verschuren et al., "Migraine and MTHFR C677T genotype in a populationbased sample," Annals of Neurology, vol. 59, no. 2, pp. 372-375, 2006.

[50] A. I. Scher, G. M. Terwindt, H. S. J. Picavet, W. M. M. Verschuren, M. D. Ferrari, and L. J. Launer, "Cardiovascular risk factors and migraine: the GEM population-based study," Neurology, vol. 64, no. 4, pp. 614-620, 2005.

[51] M. E. Bigal, T. Kurth, N. Santanello et al., "Migraine and cardiovascular disease: a population-based study," Neurology, vol. 74, no. 8, pp. 628-635, 2010.
[52] J. Schwaiger, S. Kiechl, H. Stockner et al., "Burden of atherosclerosis and risk of venous thromboembolism in patients with migraine," Neurology, vol. 71, no. 12, pp. 937948, 2008.

[53] G. C. Hall, M. M. Brown, J. Mo, and K. D. MacRae, “Triptans in migraine: the risks of stroke, cardiovascular disease, and death in practice," Neurology, vol. 62, no. 4, pp. 563-568, 2004.

[54] P. Velentgas, J. A. Cole, J. Mo, C. R. Sikes, and A. M. Walker, "Severe vascular events in migraine patients," Headache, vol. 44, no. 7, pp. 642-651, 2004.

[55] E. A. Wammes-Van Der Heijden, H. Rahimtoola, H. G. M. Leufkens, C. C. Tijssen, and A. C. G. Egberts, "Risk of ischemic complications related to the intensity of triptan and ergotamine use," Neurology, vol. 67, no. 7, pp. 1128-1134, 2006.

[56] E. Rubino, M. Ferrero, I. Rainero, E. Binello, G. Vaula, and L. Pinessi, "Association of the C677T polymorphism in the MTHFR gene with migraine: a meta-analysis," Cephalalgia, vol. 29, no. 8, pp. 818-825, 2009.

[57] J. P. Casas, L. E. Bautista, L. Smeeth, P. Sharma, and A. D. Hingorani, "Homocysteine and stroke: evidence on a causal link from mendelian randomisation," The Lancet, vol. 365, no. 9455, pp. 224-232, 2005.

[58] A. Pezzini, M. Grassi, E. Del Zotto et al., "Migraine mediates the influence of C677T MTHFR genotypes on ischemic stroke risk with a stroke-subtype effect," Stroke, vol. 38, no. 12, pp. 3145-3151, 2007.

[59] M. Schürks, R. Y. L. Zee, J. E. Buring, and T. Kurth, "Interrelationships among the MTHFR 677C > T polymorphism, migraine, and cardiovascular disease," Neurology, vol. 71, no. 7, pp. 505-513, 2008.

[60] A. Joutel, C. Corpechot, A. Ducros et al., "Notch3 mutations in CADASIL, a hereditary adult-onset condition causing stroke and dementia," Nature, vol. 383, no. 6602, pp. 707-710, 1996.

[61] H. Chabriat, K. Vahedi, M. T. Iba-Zizen et al., "Clinical spectrum of CADASIL: a study of 7 families," The Lancet, vol. 346, no. 8980, pp. 934-939, 1995.

[62] M. Verin, Y. Rolland, F. Landgraf et al., "New phenotype of the cerebral autosomal dominant arteriopathy mapped to chromosome 19: migraine as the prominent clinical feature," Journal of Neurology Neurosurgery and Psychiatry, vol. 59, no. 6, pp. 579-585, 1995.

[63] K. Vahedi, H. Chabriat, C. Levy, A. Joutel, E. TournierLasserve, and M. G. Bousser, "Migraine with aura and brain magnetic resonance imaging abnormalities in patients with CADASIL," Archives of Neurology, vol. 61, no. 8, pp. 12371240, 2004.

[64] A. E. Guttmacher, D. A. Marchuk, and R. I. White, "Hereditary hemorrhagic telangiectasia," The New England Journal of Medicine, vol. 333, no. 14, pp. 918-924, 1995. 


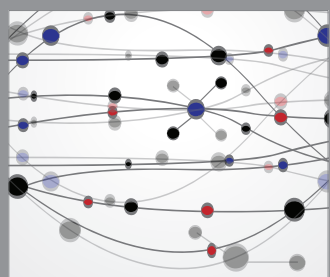

The Scientific World Journal
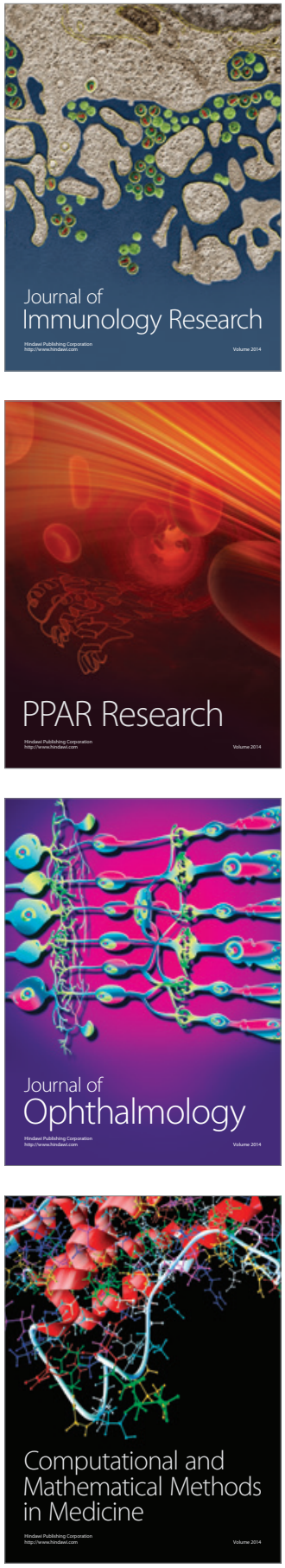

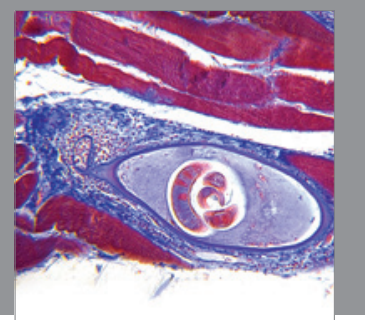

Gastroenterology

Research and Practice
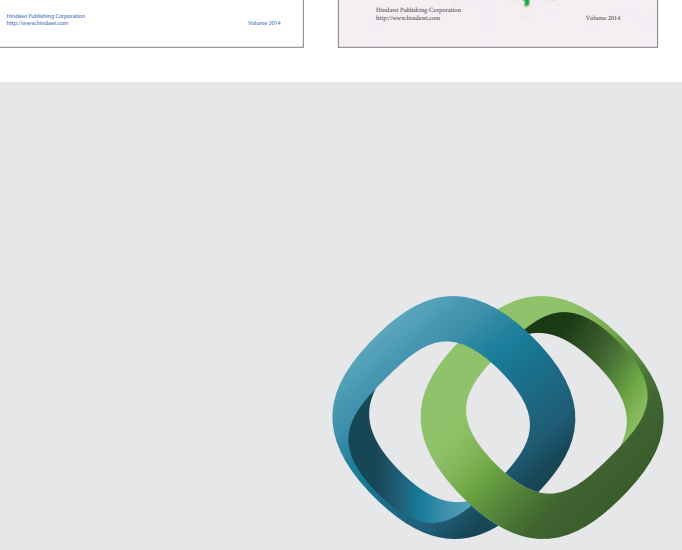

\section{Hindawi}

Submit your manuscripts at

http://www.hindawi.com
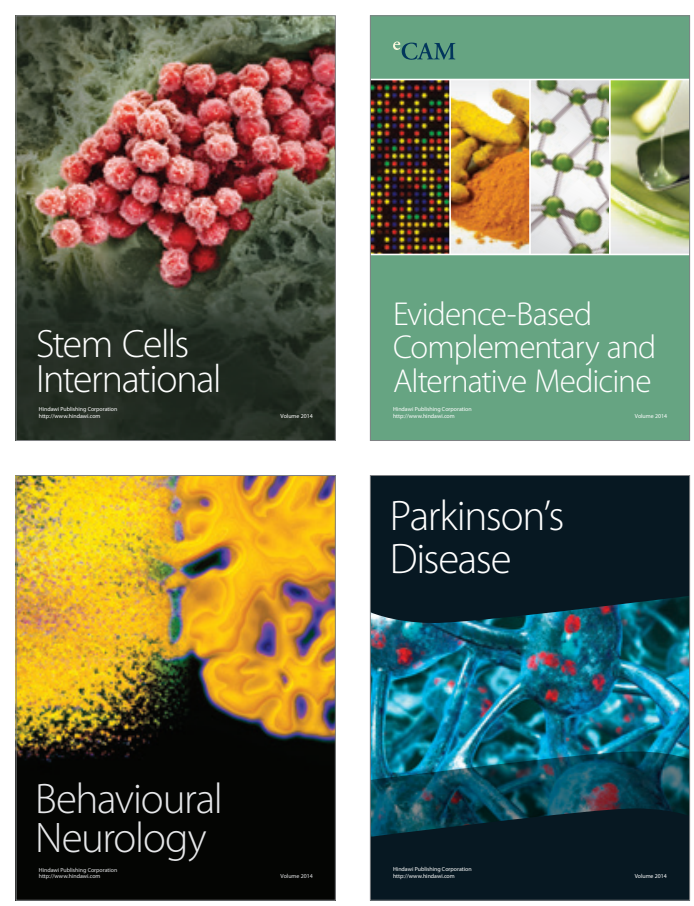

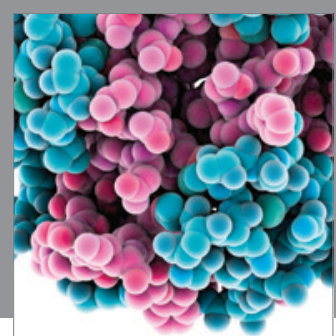

Journal of
Diabetes Research

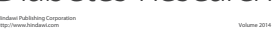

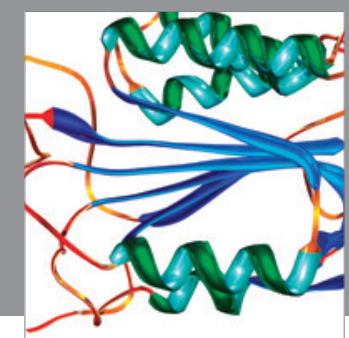

Disease Markers
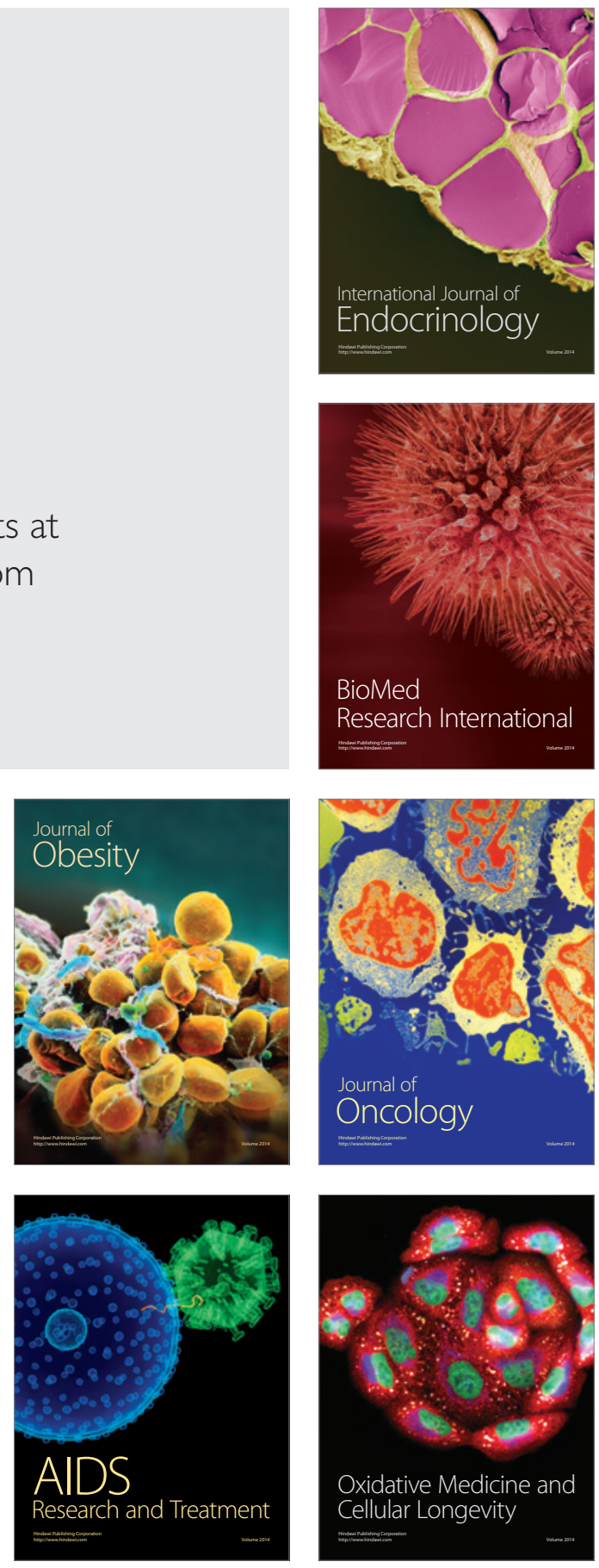